\title{
Differences in DNA content between two chromosomal races of the grasshopper Podisma pedestris
}

\author{
M. Westerman,* \\ N. H. Barton $\dagger$ and \\ G. M. Hewitt
}

\author{
* Department of Genetics and Human Variation, \\ La Trobe University, Bundoora, Victoria, \\ Australia 3083 \\ $\dagger$ Department of Genetics and Biometry, \\ University College London, Wolfson House, \\ 4 Stephenson Way, London NW1 $2 \mathrm{HE}$ \\ \$ School of Biological Sciences, \\ University of East Anglia, Norwich NR4 7TJ
}

The grasshopper Podisma pedestris contains two chromosomal races, which differ by a Robertsonian fusion between the sex chromosome and an autosome, and which meet in a narrow hybrid zone in the Alpes Maritimes. DNA content variation across this hybrid zone was investigated by optical densitometry of Feulgen stained spermatids. Spermatids from males with the unfused sex chromosome stain more strongly than those from males with the fused chromosome. The difference between the karyotypes is greater in the centre of the hybrid zone, suggesting that it is not a pleiotropic effect of the fusion itself, but is due instead to differences at closely linked loci.

\section{INTRODUCTION}

The grasshopper Podisma pedestris contains two distinct karyotypes. Over most of its range, through the Pyrenees, Appennines, and northern Alps, it has an $\mathrm{X} 0$ sex determining system. However, in the Alpes Maritimes in south-east France, a Robertsonian fusion between the $\mathrm{X}$ chromosome and an autosome has established a neo-XY system (Hewitt and John, 1972; John and Hewitt, 1970). These two chromosomal races meet in a narrow band of hybridisation which runs for $100 \mathrm{~km}$ along a high ridge of mountains near the French-Italian border (Hewitt, 1975).

Although the races were first distinguished through this chromosomal change, it is only a minor component of the differences between them. The chromosomal cline is much wider than the dispersal range of these grasshoppers $(800 \mathrm{~m}$ cf. $20 \mathrm{~m}$ ), implying that it is maintained by weak selection $(\approx 0.5$ per cent; Barton and Hewitt, $1981 a$ ). However, F1 hybrids are substantially less viable than their parental controls both as embryos and as young nymphs. Grasshoppers from the centre of the hybrid zone are even less viable than the F1s. The region over which viability is reduced is narrower than the chromosomal cline $(350 \mathrm{~m}$ cf. $800 \mathrm{~m})$, but is still much wider than the dispersal range. This implies that the inviability cannot result from strong selection at a single locus, but is due instead to incompatibilities at many loci, each of which is only weakly selected (Barton, 1980; Barton and Hewitt, $1981 b$ ).

Despite this hybrid inviability, no differences have been found at 21 enzyme loci investigated in an electrophoretic survey (Halliday, Barton and Hewitt, 1983). However, enzyme loci are not necessarily representative of the whole genome, and may not be important in evolutionary divergence. As part of a survey of other characters, therefore, we measured the DNA content of individuals collected from around the hybrid zone, by optical densitometry of Feulgen stained spermatids.

Preliminary observations showed that $\mathrm{X} 0$ males gave values $\approx 5$ per cent higher than $X Y$ males from the same population. This could be because the $\mathrm{X}$-autosome fusion involved the loss of some material from the ends of the chromosomes involved, or, conversely, because the $\mathrm{X} 0$ race had accumulated material on the $\mathrm{X}$ and/or the autosome. Any change concentrated at one point on the chromosome should be detectable cytologically; the difference in staining between the karyotypes is equivalent (judging by the length of the chromosomes at metaphase) to $\approx 50$ per cent of the whole $\mathrm{X}$ chromosome. 
Giemsa staining does reveal differences between the karyotypes of the sex chromosome races (fig. 1). The metacentric XA chromosome has an asymmetrical C-banding pattern, suggesting loss of centromeric heterochromatin from the $\mathrm{X}$ arm at or after the fusion event (fig. 2, and Westerman and Hewitt, 1985). The reduced C-band content of the chromosome race which carries the fusion is thus consistent with the direction of the staining difference observed. However, it is not sufficient to explain the magnitude of that difference.

Another possible explanation is that the two races differ in DNA content at one or many loci, and that the difference between the karyotypes is due to linkage disequilibrium with these loci. Since strong disequilibria are to be expected within hybrid zones ( $\mathrm{Li}$ and Nei, 1974; Barton, 1983), and are in fact observed in many cases (e.g.,
Uroderma bilobatum, Baker, 1981; Greenbaum, 1981; Caledia captiva, Shaw et al., 1981; Sceloporus grammicus; Hall and Selander, 1973; Bombina bombina, Szymura and Barton, 1986), and since DNA content could, in principle, change at any locus in the genome, this hypothesis is plausible. Fortunately, there is a simple method by which the action of linked loci can be distinguished from the direct pleiotropic effect of the chromosomal locus itself.

If the difference in DNA content between the karyotypes is due to disequilibria with linked loci, rather than being an intrinsic feature of the chromosomal fusion, then the difference should be greater in the middle of the zone than at the edges (fig. 3(a)). This is because at the edges, the rarer karyotype will have passed right through the hybrid zone, and so recombination will have had longer to strip away associated differences than at

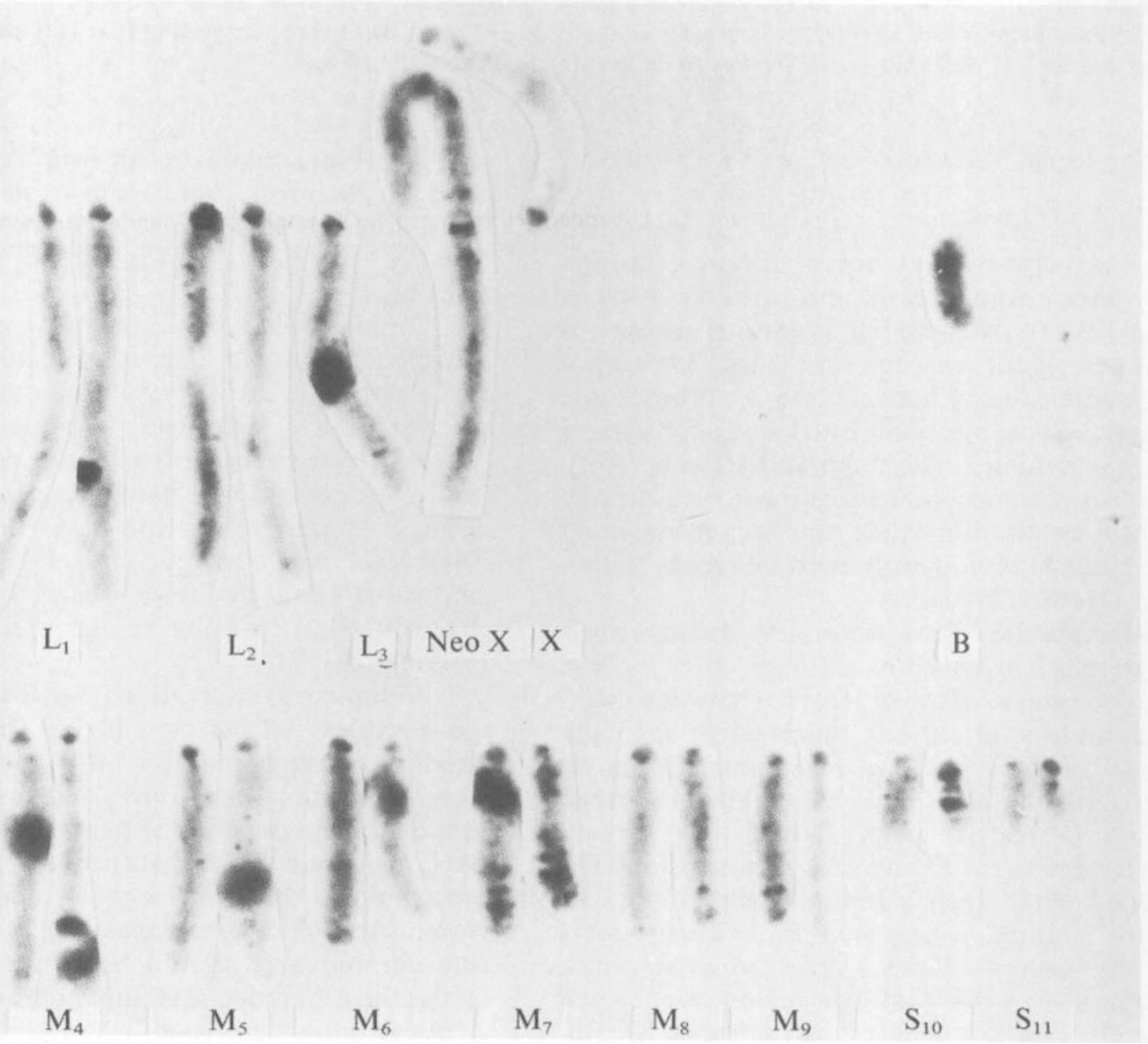

Figure $1 \mathrm{C}$ banded karyotype of a heterozygous female (neo-X, X). 

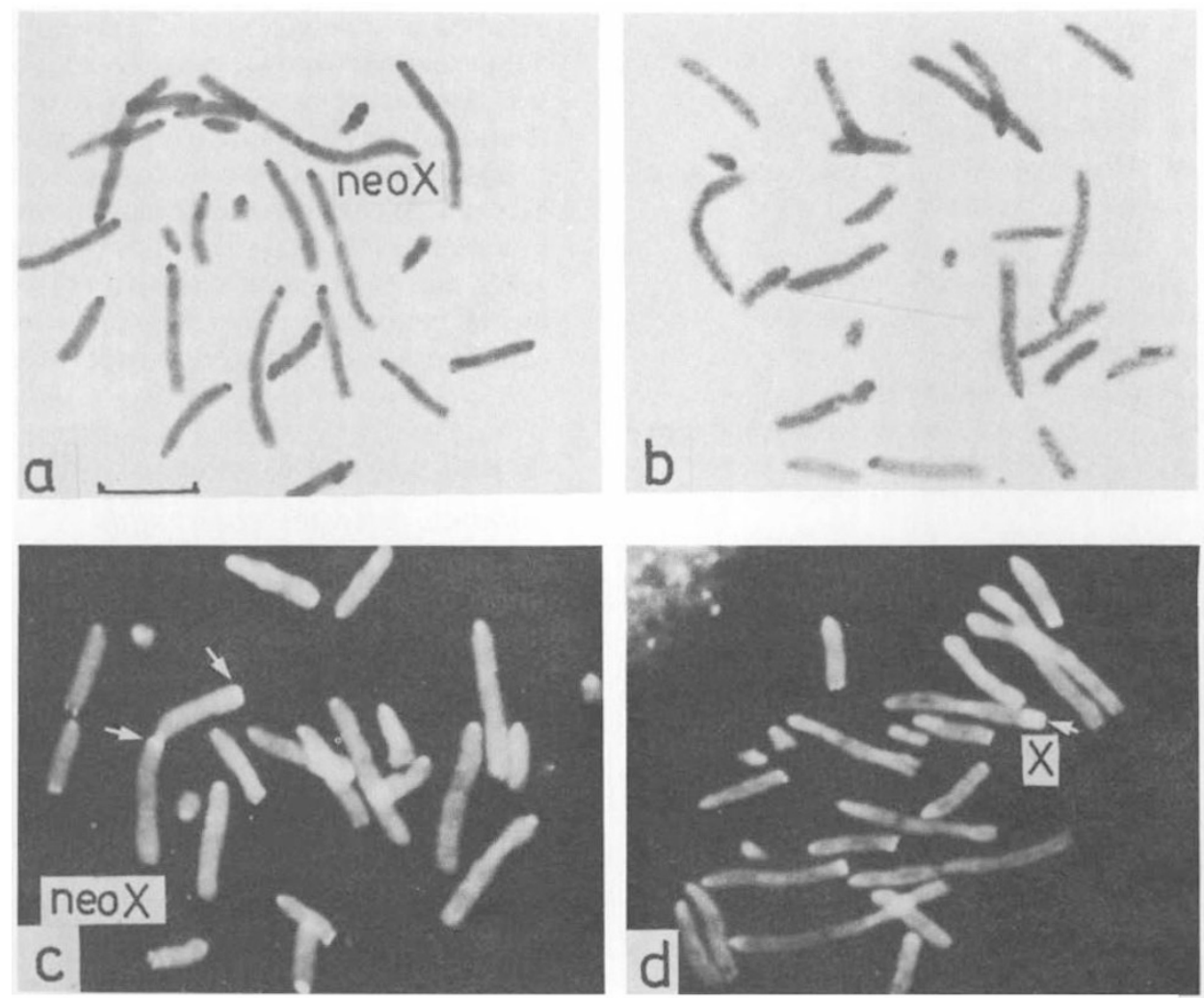

Figure 2 Banding patterns in Podisma pedestris: (a) C-banded cell from neo-X, X female, (b) C-banded cell from XX female, (c) Chromomycin $\mathrm{A}_{3}$ fluorescence of neo-X, X female (incomplete cell). Note asymmetry of fluorescence at centromere and bright fluorescence at distal end of $\mathrm{X}$ arm of the neoX. (d) Chromomycin $\mathrm{A}_{3}$ fluorescence pattern of X0 male. Note large bright fluorescent band at distal end of $X$ (arrow). (Line $=10 \mu \mathrm{m})$.

the centre, where the two genotypes first meet. Putting this mathematically, if there are a series of loci (labelled $i$ ), each with effect $\varepsilon_{i}$ and in disequilibrium $D_{i k}$ with the chromosomal locus $(k)$, the difference between the karyotypes is:

$$
\sum_{i} \varepsilon_{i} D_{i k} / p_{k} q_{k}
$$

(where $p_{k}$ is the karyotype frequency).

When recombination is loose,

$$
D_{i k} \approx \sigma^{2} p_{i}^{\prime} p_{k}^{\prime} / r_{i k}
$$

(Here, $\sigma^{2}$ is the dispersal rate, defined as variance in distance between parent and

$$
\text { offspring), }
$$

( $p_{i}^{\prime} \equiv d p_{i} / d x$ is the spatial gradient of allele frequency at locus $i$, and $r_{i k}$ is the rate of recombination between the chromosomal fusion and locus $i$; Barton, 1983). For a smooth cline such as that in Podisma, $p^{\prime}$ is proportional to $p q$. The disequilibrium therefore falls away with $(p q)^{2}$, rather than with $(p q)$ (see Barton, 1983, for a fuller discussion of the effects of linkage disequilibria in clines). Thus, we expect the difference between the karyotypes to be proportional to $(p q)$ if it is due to disequilibrium with linked loci, but to be constant if it is an inherent property of the chromosomal change (fig. 3(b)).

In order to distinguish these hypotheses, we designed an experiment to measure the difference in DNA content between males carrying the two different chromosome arrangements.

\section{METHODS AND DESIGN}

Samples were taken from the entire width of the hybrid zone, ranging from those which were almost entirely neo-XY to those which were almost entirely X0. Most samples came from the easternmost $3 \mathrm{~km}$ of the zone, near Tende, but one population (code 211) was taken from the western end, $100 \mathrm{~km}$ away near Seyne. In the statistical analysis, the positions of the samples relative to the chromosomal cline are represented by the expected 


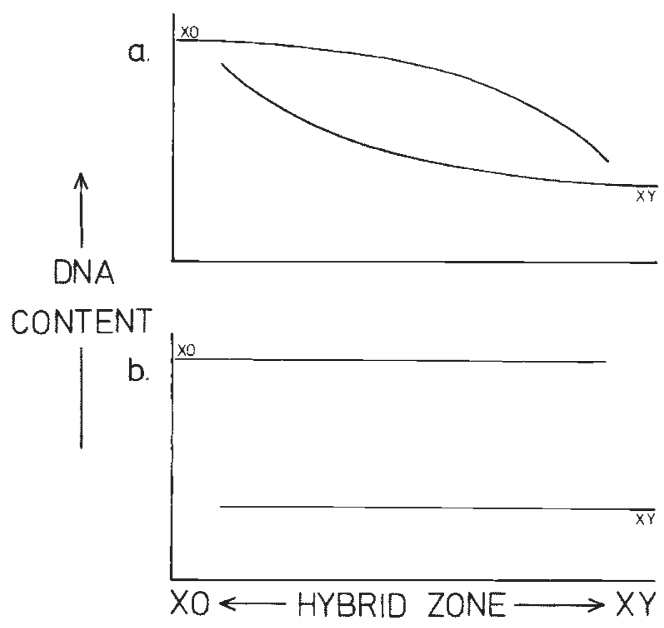

Figure 3 Expected distribution of nuclear DNA content in $\mathrm{X} 0$ and $\mathrm{XY}$ individuals across the hybrid zone if the difference in DNA content is due to (a) the effect of linked loci, or (b) the direct pleiotropic effect of the chromosomal fusion.

frequency of the fused chromosome $(p)$. This was estimated by finding the most likely cline, given all available karyotype data (2727 males at Tende, 1500 at Seyne). Although this expected frequency is subject to some sampling error, this error is negligible compared with errors in measuring the intensity of Feulgen staining. The standard error of $p$ varies from place to place, but is never more than $0 \cdot 06$. Of the 23 samples, 9 (codes 401 to 457 ) were collected during the summer of 1980 . The remainder came from earlier collections. All testes had been fixed in 1:3 acetic acid: ethanol and then stored at $4^{\circ} \mathrm{C}$.

Every male in each sample was karyotyped to establish its sex chromosome constitution, using the methods described by Hewitt, 1975. Equal numbers of $\mathrm{X} 0$ and neo-XY individuals were then chosen for the DNA measurements. All measurements were carried out using the same batches of reagents. Prior to scoring each experiment, all slides were coded and randomized so that measurements were made without knowing the sex chromosome type of the individual.

In each measurement, three or four follicles were removed from each testis, hydrolysed in $1 \mathrm{~N}$ $\mathrm{HCl}$ at $60^{\circ} \mathrm{C}$ for 10 minutes, rinsed and then stained in Feulgen reagent $(p \mathrm{H} \mathrm{3.6)}$ ) for 1 hour. Squash preparations were made in glycerol using a size 0 coverslip, sealed with rubber solution, and then scanned under a Vickers M85 microdensitometer. Spermatid nuclei which were just beginning the transition into spermatozoa were used for measurement. Ten such $1 \mathrm{C}$ nuclei per individual were scanned at a wavelength of $565 \mathrm{~nm}$, each nucleus being scanned three times. The area of the nucleus was also recorded. In all cases two or three follicles from the testis of a single male locust (Schistocerca gregaria) were used to act as an internal control. This standardises the readings across experiments. Taking the DNA content of $S$. gregaria to be $2 \mathrm{C}=$ $17.42 \mathrm{pg}$ (Wilmore and Brown, 1975), all measurements can be expressed in picograms of DNA per cell. The results are summarised in table 1.

\section{RESULTS AND ANALYSIS}

\section{Variation between spermatids}

Since the statistical analysis of the differences between $\mathrm{X} 0$ and $\mathrm{XY}$ grasshoppers depends heavil: on the degree of variation between the measurements, we deal with this aspect of the data first. There is no evidence of any variation between individuals of the same karyotype within a population, over and above that arising from variation between spermatids within individuals. The average variance between individuals with the same karyotype and from the same population is 0.786 (picograms) ${ }^{2}$, with $70 \mathrm{df}$. This may be compared with an average variance between spermatids, within individuals, of $8.617(\mathrm{pg})^{2}$, with $1044 \mathrm{df}$. Since 10 measurements were taken on each insect, the between individual variance is close to, and in fact slightly less than, that expected from sampling error $\left(F_{1044,70}=(8 \cdot 617 / 10) /(0 \cdot 786)=\right.$ $1 \cdot 09$, n.s.). There is no significant difference in between-spermatid variance between individuals within sites: Bartlett's $\chi_{93}^{2}=110 \cdot 25$, n.s., pooled across all sites. In all subsequent analysis, therefore, we estimate the variance between spermatids by pooling the data over all individuals of the same karyotype, and from the same population.

Figure 4 shows this between-spermatid variance, plotted against the expected chromosomal heterozygosity. In samples from the edge of the chromosomal cline, the standard deviation between spermatids is roughly 7 per cent of the mean. Much of this variation must be due to segregation of the $\mathrm{X}$ chromosome. Judging by chromosome lengths at diplotene, the $X$ makes up about 10 per cent of the haploid genome; its segregation will therefore contribute a standard deviation of $\approx 5$ per cent.

Variance appears higher near the centre of the hybrid zone. Dividing the populations into two sets which contribute roughly equal numbers of degrees of freedom to the variance estimate $(2 p q<$ $0.43 ; 566 \mathrm{df} ; 2 p q>0.43 ; 548 \mathrm{df})$, the ratio of the 
variance in the more central populations to the variance of those nearer the edge is $F_{548,566}=$ $11 \cdot 64 / 5 \cdot 60=2 \cdot 08$, a highly significant value. However, this test greatly exaggerates the significance of the pattern, since there are highly significant differences in variance between populations (Bartlett's test gives $\chi_{5}^{2}=97.51$ for the central populations, and $\chi_{16}^{2}=59 \cdot 13$ for the marginal populations). The unweighted regression of between-spermatid variance against the expected chromosomal heterozygosity is marginally significant $\left(\mathrm{F}_{1,21}=2 \cdot 92, p=5\right.$ per cent; we use a onetailed test here since the a priori expectation is that hybrid populations will be more variable). Kendall's rank correlation coefficient gives $\gamma=0.211$ ( $p=9$ per cent on a one-tailed test). The data no more than suggest an increased variability in hybrid populations.

The results of the following analysis of mean staining intensity depend on the assumptions made about the variance in each sample: these variances must be estimated in order to give appropriate weight to information from these samples. We have seen that there is no evidence of any variation between individuals, above that due to variation between spermatids. We can therefore estimate the variance between spermatids by pooling all measurements within each population and each chromosomal class; these are the values given in table 1 . The variance of the value measured for each individual is estimated as $1 / 10$ of this value, since it is based on 10 spermatids.

A second problem is associated with differences in variance between populations. One possibility would be to base the following analysis on the average variance over the whole transect; this would clearly be misleading, since there is considerable heterogeneity between samples. Instead, we will use the observed variance as an estimate of the actual variance in each separate sample.

Table 1 Summary of data from 23 populations. Population code: all samples are from the eastern $2 \mathrm{~km}$ of the hybrid zone, near Col de Tende, except for 211 , which is from Seyne, $100 \mathrm{~km}$ to the west at the opposite end of the zone. Per cent XY: the expected proportion of XY karyotypes, estimated by fitting a smooth cline to all available karyotype data. Sample size: the numbers used in this analysis; equal numbers of X0 and XY were used, except for $101(2 \mathrm{X} 0,1 \mathrm{XY})$ and 123 (1 X0, $2 \mathrm{XY}$ ). Mean: the overall mean for each sample. All values are estimated $2 C$ picograms of DNA, calibrated using $S$. gregaria $2 \mathrm{C}=17.42 \mathrm{pg}$. Difference: the difference between the means of $\mathrm{X} 0$ and $\mathrm{XY}$ males. Variance: the variance in DNA content of each spermatid about the mean for each separate karyotype in each population. (Units are $\left(2 \mathrm{C}\right.$ picograms DNA $\left.{ }^{2}\right) t, \mathrm{df}$ : one-tailed $t$-tests for significance of the difference between karyotypes; these are based on the variance between spermatids given in the preceding column

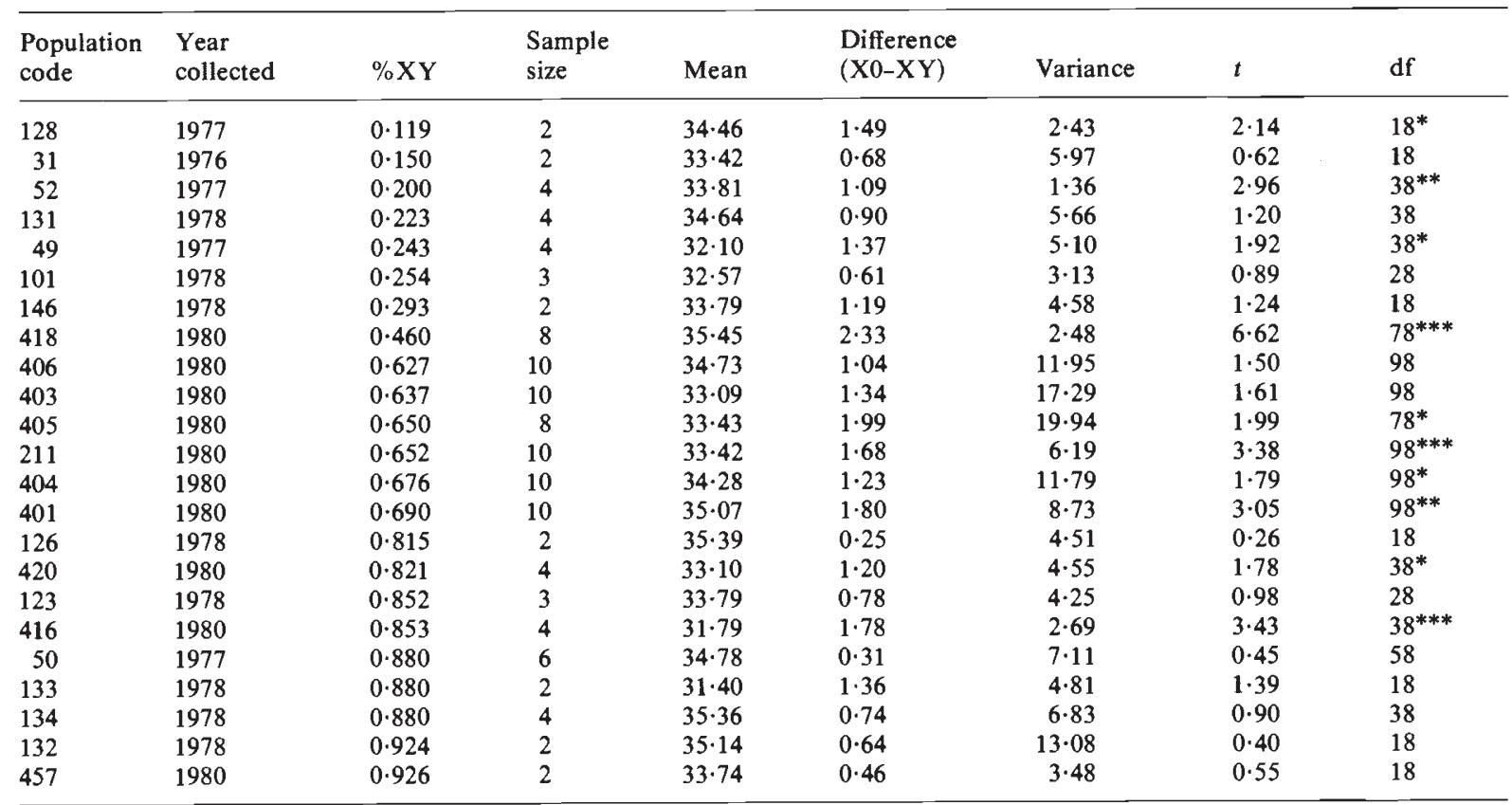




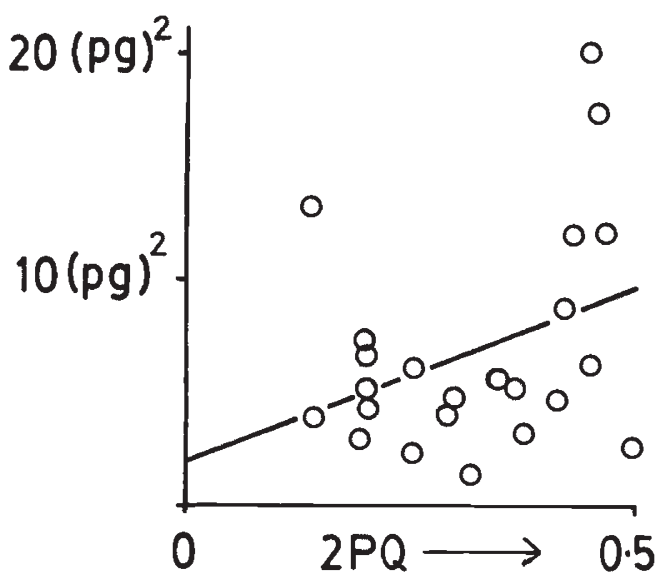

Figure 4 The average variance between spermatids (calculated by pooling over all individuals with the same karyotype, and from the same population), plotted against the expected chromosomal heterozygosity ( $2 \mathrm{pq}$ ) for each of 23 populations. The variance is given in $(2 \mathrm{C} \text { picograms DNA })^{2}$, derived by calibration against $S$. gregaria (Wilmore and Brown, 1975). The line shows the most likely regression of variance against heterozygosity; each population was given equal weight in calculating this regression.

\section{Differences between karyotypes}

The X0 males have more DNA than the XY's in all 22 populations from Tende, and also in the one sample taken from Seyne, $100 \mathrm{~km}$ along the hybrid zone to the west. Of the 23 one-tailed $t$-tests calculated for each population, 10 are significant at the 5 per cent level, and of these 5 are significant at the 1 per cent level (table 1). Crudely, the mean $2 \mathrm{C}$ value of $\mathrm{X} 0$ males is $34.8 \mathrm{pg}$, compared with $33.2 \mathrm{pg}$ for XY males, a 4.7 per cent difference. This could be an intrinsic effect of the chromosomal rearrangement, or it could be due to disequilibrium with closely linked loci. As discussed above, we can distinguish these possibilities by plotting the difference between karyotypes against expected chromosomal heterozygosity (fig. 5). The difference does seem greater in the centre; the regression line obtained by weighting each population by the estimated variance runs from a $0 \cdot 1 \mathrm{pg}$ difference at the edge up to a $1.8 \mathrm{pg}$ difference in the centre. If the variance accounted for by this fit is compared with the residual variance, the result is highly significant $\left(\mathrm{F}_{1,21}=15 \cdot 16, p<0 \cdot 1\right.$ per cent in a one-sided test). However, we should point out that the residual variance is a good deal less than would be expected from the variance between spermatids within each sample $\left(\chi_{21}^{2}=10.97, p \approx 5\right.$ per cent). When the variance accounted for by the regression is compared with the residual expected from the between spermatid variance, it is still significant, though less so $\left(\chi_{1}^{2}=7 \cdot 92, p \approx 0.5\right.$. per cent in a one-sided test).

\section{Differences between the races}

As well as looking for differences between karyotypes within mixed populations, we can also look for differences between populations on either side of the cline. This is more difficult, for two reasons. First, the experiment was designed to allow a controlled comparison between samples from the same population; measurement errors between populations scored on different days may be larger. Second, the means given in table 1 are the means of the insects actually measured, and are a biased estimate of the population mean. This is because equal numbers of insects of each karyotype were chosen, whereas the populations, in fact, had widely varying proportions of the karyotypes. The values used in thie following regression (fig. 6) have therefore been corrected on the basis of the estimated difference between karyotypes to give an estimate of the true population mean. (A term $\left(p_{\text {population }}-p_{\text {sample }}\right) \Delta$ was added, where $p$ is the frequency of the XY karyotype (estimated from the fitted cline), and $\Delta$ the difference in staining between $\mathrm{XY}$ and $\mathrm{X} 0$ derived from the regression in fig. 5. Each population has been weighted according to the estimated between spermatid variance, as before.

The difference between the races is estimated as $(\mathrm{X} 0-\mathrm{XY})=1.4 \mathrm{pg}$, with 95 per cent confidence limits $(-0.6$ to $+3.4 \mathrm{pg}$ ) (fig. 6) (Note that this estimate must not be confused with the difference between karyotypes within populations, estimated above). The residual variation between populations is much greater than that expected from the within-population variance $\left(\chi_{21}^{2}=286 \cdot 36, p<0 \cdot 1\right.$ per cent). Thus, the overall difference between the insects on either side of the cline is of the same order as that between karyotypes within the zone, and is certainly not much greater. This suggests that if linkage disequilibrium is responsible for the $\approx 1.8 \mathrm{pg}$ difference between karyotypes within central populations, then disequilibrium must be strong: most of the effect must be due to loci closely linked to the chromosomal fusion.

\section{DISCUSSION}

There is a large and consistent difference in mean Feulgen staining between $\mathrm{X} 0$ and $\mathrm{XY}$ males taken from within the same population. The mapping of the difference across the hybrid zone shows that 


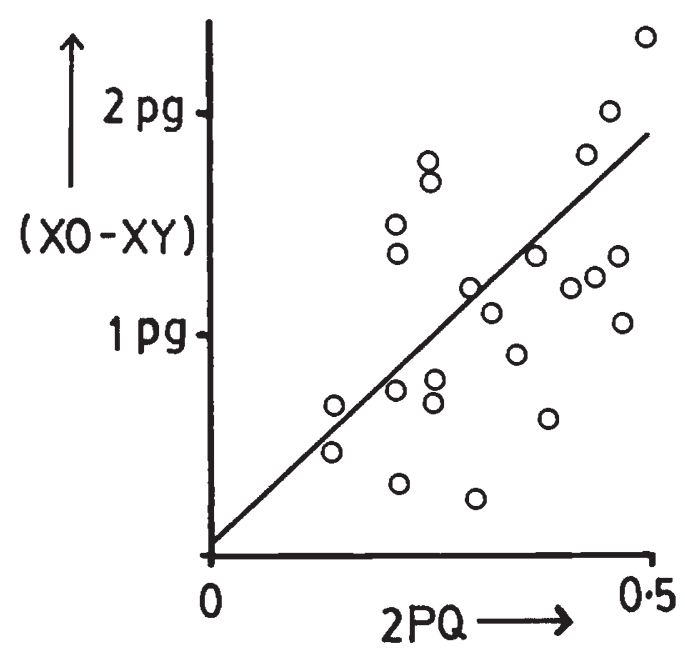

Figure 5 The average difference between $X_{0}$ and $X Y$ individuals (in $2 \mathrm{C}$ picograms DNA), plotted against the expected chromosomal heterozygosity, for each of 23 populations. The line shows the most likely regression between the two variables; each population is given weight equal to the inverse of the sampling error expected to arise from the variance between spermatids.

it is greater in central populations, $(1 \cdot 8 \mathrm{pg}(\approx 5 \cdot 3$ per cent), compared with $0 \cdot 1 \mathrm{pg}(\approx 0 \cdot 2$ per cent $))$. It is therefore more likely that it is caused by linkage disequilibrium with one or more distinct loci than that it is an intrinsic pleiotropic effect of the Robertsonian fusion. The loci responsible for the difference must recombine with the fusion at a rate $>0.5$ per cent. This figure is the estimated selection pressure acting on the chromosomal difference (Barton and Hewitt, 1981 $a$ ), and gives a limit to the resolution of our method: any loci more closely linked than 0.5 per cent will not be distinguishable from a purely pleiotropic effect. However, these loci (or this locus) must nevertheless be in quite strong disequilibrium with the fusion, since the difference between the races on either side of the cline was not much greater than that difference between karyotypes ( 95 per cent confidence limit $<3 \cdot 4 \mathrm{pg}$ ).

If the difference in staining is due to differences in DNA content, it is hard to see how such a large effect could be concentrated into a restricted region of the chromosome without causing a very much larger difference in size and $\mathrm{C}$ banding than that observed. An alternative possibility is that the karyotypes differ in condensation of the chromatin, rather than in actual DNA content. The pattern of variances also bears on this distinction. No significant variation in staining was found between individuals, despite a substantial variance between spermatids. The variance between spermatids can- not therefore be passed on to generate variance between individuals in the next generation. This could be because there is very strong stabilising selection on the sperm: one might investigate this hypothesis by making measurements at various stages from meiosis through the embryos and into adult tissues. Alternatively, if the variance is due to differing degrees of condensation, then it may simply not be heritable. (It is unlikely that the variance reflects measurement error, since there is substantial heterogeneity in variance between sites, and since the variance seems greater in hybrid populations).

We may be able to distinguish these two interpretations by considering the measurements of spermatid area, as well as the total staining intensity. There is a strong correlation between total Feulgen staining, and the area of each spermatid $(r=0.55,115 \mathrm{df} ; p<0.1$ per cent). This could be because differential condensation affects the apparent total intensity or because differences in total DNA content affect the apparent (or actual) area. The area measurements show the same pattern as the total Feulgen staining, but less strongly: the ratio between the within-individual variances in area for the six most hybrid populations, and the remainder, is only $1 \cdot 27$. This compares with a ratio of 2.05 for Feulgen staining. Similarly, the X0 spermatids have only $2 \cdot 7$ per cent greater area measure than the $\mathrm{XY}$, as compared with a $4 \cdot 2$ per cent difference for the total Feulgen staining. (These figures differ slightly from those in the rest of the analysis, since they are calculated without weighting the populations differently). This suggests, but does not prove, that the difference between hybrid and non-hybrid, and

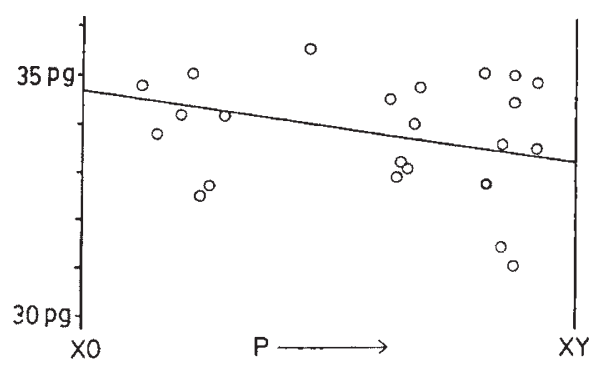

Figure 6 The mean DNA content for each population (in $2 \mathrm{C}$ picograms DNA), after correction for the component associated with the chromosomal fusion (see text), plotted against the expected frequency of the fusion $(p)$. The line shows the regression of mean DNA content against $p$; each population is given weight equal to the inverse of the sampling error expected to arise from the variance between spermatids. 
between $\mathrm{X} 0$ and $\mathrm{XY}$, is due to a difference in DNA content rather than in condensation.

There is a suggestion that the variance between spermatids is greater at the centre of the hybrid zone (fig. 4). Though marginally significant, this pattern is analogous to other phenomena in Podisma: there is a 5-fold increase in the frequency of rare electrophoretic variants at two loci (Barton, Halliday and Hewitt, 1983), and a 2-fold increase in the frequency of chromosomal aberrations in hybrid embryos (Barton, 1980) (though the overall frequency of these latter aberrations is much too low to account for the variation in Feulgen staining). Such phenomena are frequently found in other hybrid zones (Barton and Hewitt, 1985).

The most important biological question raised by these results is their relation to the $\approx 50$ per cent reduction in hatchability observed in hybrids (Barton, 1980; Barton and Hewitt, $1981 b$ ), and thus to the development of isolation between the two diverging taxa. The variance between spermatids suggests that some of the reduction in hatchability may be due to male sterility. However, three lines of evidence indicate that the variation between spermatids is unlikely to be responsible for all or even most of the mortality. First, there is a reduced hatch rate in F1's from crosses between pure populations, and there is no consistent difference between reciprocal crosses. Second, although the proportion of haploid pre-diapause embryos is somewhat increased in hybrids, it is still not high (Barton, 1980), suggesting that failure of fertilisation does not cause much mortality. Third, there is significant mortality in the first week of nymphal life, as well as at all the preceding stages of embryonic development (Barton and Hewitt, 1981b, and unpublished results). As for the effect of the difference between karyotypes on fitness, we can say that it must be slight $(\approx 0.5$ per cent $)$, simply because the chromosomal cline is wide compared with the dispersal range of this grasshopper $(\approx 800 \mathrm{~m} \mathrm{cf} . \approx 20 \mathrm{~m})$. On the assumption that the difference is due to changes in DNA content of $\simeq 5$ per cent, this weak selection may seem surprising. However, the effects of similar changes in DNA content in other organisms have been found to be slight (e.g. Hewitt, 1973; Rees, 1982).

\footnotetext{
Acknowledgements We would like to thank Marise East for technical assistance and M. et Mme Aviotti for their hospitality in France. We are grateful to Dr Michael Bennet, Dr Donald Fox, Professor Hubert Rees, and an anonymous referee for their helpful comments on earlier manuscripts. This work was supported by an S.E.R.C. grant to G.M.H., and by an S.E.R.C. postdoctoral fellowship to N.H.B. M.W. worked at UEA under an Outside Studies Program.
}

\section{REFERENCES}

BAKER, R. J. 1981. Chromosome flow between chromosomally identified taxa of a volant mammal, Uroderma bilobatum (Chiroptera: Phyllostomatidae). Evolution, 35, 296-305.

BARTON, N. H. 1980. The fitness of hybrids between two chromosomal races of the grasshopper Podisma pedestris. Heredity, 45, 47-59.

BARTON, N. H. 1983. Multilocus clines. Evolution, 37, 454-471. BARTON, N. H., HALlidAY, R. B. AND HEWITT, G. M. 1983. Rare electrophoretic variants in a hybrid zone. Heredity, $50,139-146$

BARTON, N. H. AND HEWITT, G. M. $1981 a$. A chromosomal cline in the grasshopper Podisma pedestris. Evolution, 35, 1008-1018.

BARTON, N. H. AND HEWITT, G. M. $1981 b$. The genetic basis of hybrid inviability between two chromosomal races of the grasshopper Podisma pedestris. Heredity, 47, 367-383.

BARTON, N. H. AND HEWITT, G. M. 1985. Analysis of hybrid zones. Ann. Rev. Ecol. Syst., 16, 113-148.

GREENBAUM, I. F. 1981. Genetic interaction between hybridising cytotypes of the tent-making bat (Uroderma bilobatum). Evolution, 35, 306-321.

HALL, W. P. AND SELANDER, R. K. 1973. Hybridisation of karyotypically differentiated populations of the Sceloporus grammicus complex (Iguanidae). Evolution, 27, 226-242.

HALlidAY, R. B., BARTON, N. H. AND HEWITT, G. M. 1983. Electrophoretic analysis of a chromosomal hybrid zone in the grasshopper Podisma pedestris. Biol. J. Linn. Soc., 19, 51-62.

HEWITT, G. M. 1973. The integration of supernumerary chromosomes into the Orthopteran genome. Cold Spring Harbor Symp. Quant. Biol., 38, 183-194.

HEWITT, G. M. 1975. A sex-chromosome hybrid zone in the grasshopper Podisma pedestris (Orthoptera: Acrididae). Heredity, 35, 375-387.

HEWITT, G. M. AND JOHN, B. 1972. Interpopulation sex chromosome polymorphism in the grasshopper Podisma pedestris. II Population parameters. Chromosoma, 37, 2342.

JOHN, B. AND HEWITT, G. M. 1970. Interpopulation sex chromosome polymorphism in the grasshopper Podisma pedestris. II Fundamental facts. Chromosoma, 31, 291-308.

LI, W. H. AND NEI, M. 1974. Stable linkage disequilibrium without epistasis in subdivided populations. Theoret. Pop. Biol., 6, 173-183.

REES, H., JENKINS, G., SEAL, A. G. AND HUTCHINSON, J. 1982. Assays of the phenotypic effects of changes in DNA amounts. Dover, G. A. and Flavell, R. B. (eds.) In Genowie Evolution, Systematics Assoc. Special Volume 20, Academic Press, London, pp. 287-297.

SHAW, D. D., MORAN, C. AND WILKINSON, P. 1981. Chromosomal reorganisation, geographic differentiation, and the mechanism of speciation in the genus Caledia. Blackman, R. L., Hewitt, G. M. and Ashburner, M. (eds.). In Insect Cytogenetics, Royal Entomol. Soc. Symp. X, Blackwells, Oxford, pp. 149-170.

SZYMURA, J. AND BARTON, N. H. 1986. Genetic analysis of a hybrid zone between the fire-bellied toads Bombina bombina and $B$. variegata, near Cracow in southern Poland. Evolution (in press)

WESTERMAN, M. AND HEWITT, G. M. 1985. Chromosone banding in Podisma pedestris. Heredity, 55, 157-161.

WILMORE, P. AND BROWN, A. K. 1975. Molecular properties of Orthopteran DNA. Chromosoma, 51, 337-345. 